\title{
Spontaneous alterations of regional brain activity in patients with adult generalized anxiety disorder
}

\author{
This article was published in the following Dove Press journal: \\ Neuropsychiatric Disease and Treatment \\ 20 July 2017 \\ Number of times this article has been viewed
}

Likun Xia'
Shumei Li'
Tianyue Wang
Yaping Guo
Lihong Meng
Yunping Feng
Yu Cui'
Fan Wang'
Jian Ma'
Guihua Jiang
'Department of Magnetic Resonance
Imaging, People's Hospital of Yuxi City,
Yuxi, 'Department of Medical Imaging,
Guangdong No 2 Provincial People's
Hospital, Guangzhou, 'Department
of Rehabilitation Medicine, People's
Hospital of Yuxi City, Yuxi, People's
Republic of China

Objective: We aimed to examine how spontaneous brain activity might be related to the pathophysiology of generalized anxiety disorder (GAD).

Patients and methods: Using resting-state functional MRI, we examined spontaneous regional brain activity in $31 \mathrm{GAD}$ patients (mean age, $36.87 \pm 9.16$ years) and 36 healthy control participants (mean age, $39.53 \pm 8.83$ years) matched for age, education, and sex from December 2014 to October 2015. We performed a two-sample $t$-test on the voxel-based analysis of the regional homogeneity (ReHo) maps. We used Pearson correlation analysis to compare scores from the Hamilton Anxiety Rating Scale, Hamilton Depression Rating Scale, State-Trait Anxiety Scale-Trait Scale, and mean ReHo values.

Results: We found abnormal spontaneous activity in multiple regions of brain in GAD patients, especially in the sensorimotor cortex and emotional regions. GAD patients showed decreased ReHo values in the right orbital middle frontal gyrus, left anterior cingulate cortex, right middle frontal gyrus, and bilateral supplementary motor areas, with increased ReHo values in the left middle temporal gyrus, left superior temporal gyrus, and right superior occipital gyrus. The ReHo value of the left middle temporal gyrus correlated positively with the Hamilton Anxiety Rating Scale scores.

Conclusion: These results suggest that altered local synchronization of spontaneous brain activity may be related to the pathophysiology of GAD.

Keywords: generalized anxiety disorder, functional magnetic resonance imaging, resting state, regional homogeneity

\section{Introduction}

Generalized anxiety disorder (GAD) is marked by excessive and uncontrollable anxiety and worry. GAD is a common anxiety disorder that shows a lifetime prevalence of $4.3 \%-5.9 \%$ and a $12-$ month prevalence of $0.2 \%-4.3 \% .^{1,2}$ GAD patients have a higher prevalence of lifetime suicidal thoughts and attempts than patients with other anxiety disorders. Currently, diagnosis of GAD is based mainly on clinical psychiatric signs and symptoms. However, such an approach might lead to misdiagnosis as a result of high rates of comorbidity with depression and agoraphobia. ${ }^{3}$ Therefore, an objective and stable method to improve the diagnostic accuracy of GAD is needed.

In recent years, researchers have used a variety of neuroimaging techniques to explore the pathogenesis of GAD. Using single-photon emission computed tomography, Lee et $\mathrm{al}^{4}$ found that the striatal dopamine transporter level in patients with GAD could have a role in the pathophysiology of GAD. Using proton magnetic resonance spectroscopy, Moon et $\mathrm{al}^{5}$ found that the choline $/ N$-acetylaspartate metabolic changes in the dorsolateral prefrontal cortex in patients with GAD were closely related to symptom severity and cognitive dysfunction. In addition, diffusion tensor imaging 
studies found that adult patients with GAD showed lower fractional anisotropy in the uncinate fasciculus, which connects the amygdala and the frontal cortex. ${ }^{6,7}$

More researchers have focused on functional magnetic resonance imaging (fMRI) technology. Evidence from various task-based fMRI studies has indicated that an alteration occurs in the activation of the anterior cingulate cortex (ACC), prefrontal cortex (PFC), insula, amygdala, and other limbic brain regions when patients with GAD perform specific tasks. ${ }^{8-11}$ Differentiation of these functional alterations might reveal abnormal neural circuit and emotion regulation in GAD patients. However, task-based fMRI requires good cooperation from subjects and can only reflect the brain neuron activities associated with the specific tasks, implying that task-based fMRI might have less clinical practicability. Therefore, to understand how the brain operates in GAD, we must consider the spontaneous neuronal activity which consumes most of the brain's energy.

The noninvasive resting-state fMRI (rs-fMRI), unlike the complex task-based fMRI, can be performed conveniently in clinics and is widely used to evaluate spontaneous neuronal activity. A systematic review of the literature showed that an increasing number of researchers have used rs-fMRI techniques and functional connectivity analysis to study other types of anxiety disorders including post-traumatic stress disorder, obsessive-compulsive disorder, social anxiety disorder, and panic disorder. ${ }^{12}$ However, regional spontaneous activity in GAD based on rs-fMRI techniques has not been extensively reported in previous studies. Moreover, the most commonly used is the method of functional connectivity in GAD based on rs-fMRI. ${ }^{13-15}$ Makovac et al ${ }^{14}$ showed reduction in connectivity between right amygdala and ventromedial PFC, enhanced coupling between left amygdala and ventral tegmental area, and increased connectivity between right amygdala and thalamus. Results highlighted amygdala functional connectivity as a longitudinal biomarker of symptom changes in GAD. Functional connectivity showed the interaction between brain regions.

Regional homogeneity ( $\mathrm{ReHo}$ ) is widely used with rs-fMRI to evaluate regional spontaneous activity across the whole brain. ReHo can measure the similarity of voxel of the time series of a given voxel with its nearest neighbors by calculating Kendall's coefficient of concordance (KCC). ReHo has been applied commonly to various neuropsychiatric disorders, such as sleep disorders, ${ }^{16}$ transient ischemic attack, ${ }^{17}$ rolandic epilepsy, ${ }^{18}$ and depression. ${ }^{19}$ To our knowledge, the ReHo approach has not been used to investigate spontaneous brain activity in GAD.
In this study, we used the ReHo method based on rs-fMRI to gain additional insights into aberrant spontaneous neural activity in GAD patients. In accordance with previous findings, we hypothesized that compared with healthy controls, patients with GAD would exhibit abnormal ReHo activity, mainly in sensorimotor cortex and emotional regions. Moreover, we speculated that the ReHo values extracted from the abnormal regions would be correlated with an emotional scale, thereby providing potential imaging evidence to explain the clinical manifestations of GAD.

\section{Patients and methods Subjects}

This study included a group of $31 \mathrm{GAD}$ patients (mean age, $36.87 \pm 9.16$ years; 15 males and 16 females). The study also included 36 healthy sex-, hand-, age-, and educationmatched controls (mean age, 39.53 \pm 8.83 years; 13 males and 23 females). The study was approved by the Research Ethics Committee of People's Hospital of Yuxi City (Yuxi, People's Republic of China). All participants signed written informed consent agreement, and participants were reimbursed $\$ 50$ for their time/efforts.

GAD patients were recruited from the Department of Rehabilitation Medicine at People's Hospital of Yuxi City from December 2014 to October 2015. Diagnosis of GAD was determined by consensus of two experienced psychiatrists. The inclusion criteria for patients with GAD were as follows: 1) conforming to the criteria of the Diagnostic and Statistical Manual of Mental Disorders, Version IV; 2) had been complaining of uncontrollable anxiety and worry about everyday events and problems for at least 6 months; 3) had not taken antianxiety or antipsychotic drugs for at least 1 month before the study; 4) had a Hamilton Anxiety Rating Scale (HAMA) score $\geq 14$; and 5) being younger than 60 years old.

The exclusion criteria for patients with GAD were as follows: 1) having other psychiatric disorders and secondary anxiety disorder; 2) exhibiting drug or alcohol dependence; 3) having had a past history of depression or episodes of depression; 4) having a 24-item Hamilton Depression Rating Scale (HAMD) score $>20 ; 5$ ) having been diagnosed with other significant medical conditions, such as diabetes or high blood pressure; 6) having an abnormal signal as verified by conventional T1 or T2 fluid-attenuated inversion recovery MRI; 7) being pregnant or breastfeeding. We excluded four GAD patients with abnormal signals in the conventional T2 image.

The inclusion criteria for healthy controls were as follows: 1) having a HAMA score $<7$ and a 24 -item HAMD 
score $<8$; 2) a conventional MRI showing no organic disease; 3 ) had no history of inborn or other psychiatric disorder; and 4) had no metal foreign objects in his/her body.

\section{Additional clinical measures}

Before MRI examinations, the emotional quantifiable indicators of every subject were rated by the HAMA and the 24-item HAMD scales as well as the State-Trait Anxiety Inventory (STAI) scale.

\section{MRI data acquisition}

All MRI datasets were acquired using a 3 T MRI scanner with an eight-channel head coil (Ingenia; Philips, the Netherlands) in the MRI Department of People's Hospital of Yuxi City. Each subject was supine with headphones to reduce noise and was positioned with foam pads to secure the head and reduce head motion. During the rs-fMRI data acquisition, all subjects were asked to keep their eyes closed, relax, hold still, not think of anything systematically, and not fall asleep. The rs-fMRI scans were performed using a gradient echo planar imaging sequence. The acquisition parameters were as follows: repeat time $=2,000 \mathrm{~ms}$; echo time $=35 \mathrm{~ms}$, flip angle $=90^{\circ}$; field of view $=230 \times 230 \mathrm{~mm}$; matrix $=64 \times 64$; total volume $=240$; voxel size: $3.6 \times 3.6 \times 3.6 \mathrm{~mm}$; slice thickness $=3.6 \mathrm{~mm}$ with a $0.7 \mathrm{~mm}$ gap; 35 transverse plane parallel AC-PC line; 240 dynamic scanning; 8,400 images. The fMRI scanning for each subject lasted 8 minutes. After the MRI scanning, the patient was interviewed to assess whether he or she had complied with the instructions and was awake throughout the scan. Subjects who failed to comply with the instructions were excluded from the study.

\section{Data processing and ReHo analyses}

Data preprocessing was based on a Matlab 2012b operation interface, using the Data Processing Assistant for RestingState fMRI integrated software (http://restfmri.net/forum/ DPARSF $).{ }^{20}$ For data processing, the first 10 time points were discarded due to transient signal changes before magnetization reached a steady state and/or adaptation of the subject to the circumstances, leaving 230 time points for the preprocessing steps of slice timing. No subject's data were discarded as a result of excessive head motion $(>1.5 \mathrm{~mm}$ in translation or $1.5^{\circ}$ in rotation).

Next, we compared the motion courses of the two groups using a two-sample $t$-test. This test confirmed no statistical significance. After head motion correction, the fMRI images were normalized to the Montreal Neurological Institute template $\left(\right.$ resampling voxel size $=3 \times 3 \times 3 \mathrm{~mm}^{3}$ ). Linear trends and temporal filtering (band pass, $0.01-0.08 \mathrm{~Hz}$ ) were removed to reduce very low frequency drift and physiologic highfrequency respiratory and cardiac noise. ${ }^{21}$

The ReHo calculation procedure used the Resting-State fMRI Data Analysis Toolkit (REST; http://resting-fmri. sourceforge.net), the same as described in previous studies. ${ }^{22}$ A KCC value (ReHo value) was used to measure the similarity of the time series of a given voxel to its nearest 26 neighbors. ${ }^{23}$ The KCC value calculation formula was as follows, where $W$ is the $\mathrm{KCC}$ value of that given voxel, ranging from 0 to 1 :

$$
W=\frac{\sum\left(R_{i}\right)^{2}-n(\bar{R})^{2}}{\frac{1}{12} K^{2}\left(n^{3}-n\right)}
$$

When a given cluster and its neighboring cluster in a time sequence are more consistent, the KCC value is close to 1 . $R_{i}$ is the sum rank of the ith time point. $\bar{R}=[(n+1) K] / 2$ is the mean of the Ris. $K$ is the number of voxels within a measured cluster (here, $K=27$, the number of nearest neighboring 26 voxels plus that one given voxel), and $n$ is the number for the ranks (here, $\mathrm{n}=150$ ).

Normalized ReHo value is the KCC value of each voxel/ the mean of the whole brain KCC. The individual ReHo map was obtained for each data set. Finally, we applied an $8 \mathrm{~mm}$ full width at half maximum spatial smoothing Gaussian kernel to reduce the spatial noise.

\section{Statistical analysis}

We used SPSS Version 20.0 (IBM Corporation, Armonk, NY, USA) for analyzing demographic data and clinical characteristics. A chi-square test was used for sex comparison. A two-sample $t$-test was performed to evaluate the differences in age, education, HAMA, HAMD, and State-Trait Anxiety Scale-Trait Scale (STAI-T) scores between patients with GAD and healthy controls, with the significance level set at $P<0.05$.

A two-sample $t$-test was also applied to compare the smReHo maps between GAD patients and healthy controls. Multiple comparison correction was performed by using the AlphaSim program in REST and a $P$-value $<0.05$. For each region of interest, the mean ReHo values for all voxels in the significant areas were extracted separately using the REST. Finally, Pearson correlation analysis was also adopted to clarify the relationship between the mean ReHo values in significantly different areas and the HAMA, HAMD-24, and STAI-T scores in SPSS $20.0(P<0.05)$. 


\section{Results}

\section{Demographic and clinical variables}

The study results demonstrated no significant differences in sex $(P=0.310)$, age $(P=0.232)$, and education $(P=0.088)$ between the GAD group and the healthy control group. The HAMA, HAMD-24, and STAI-T scores of the GAD subjects were significantly higher than those of the healthy controls. The detailed demographic and clinical data are presented in Table 1.

\section{Alterations in ReHo values of GAD patients}

In this study, GAD patients showed significantly different ReHo values in widespread areas when compared with healthy controls (voxel $P<0.05$, cluster $P<0.05$ with AlphaSim corrected). The detailed between-group differences of ReHo values are presented in Table 2 and Figures 1 and 2. Compared to the healthy controls, GAD patients had significantly decreased ReHo values in the right orbital middle frontal gyrus (MFG), left ACC, right MFG, and bilateral supplementary motor area (SMA). GAD patients showed increased ReHo values in the left middle temporal gyrus (MTG), left superior temporal gyrus (STG), and right superior occipital gyrus (SOG).

\section{The correlation between ReHo values and clinical variables in GAD patients}

As shown in Figure 3A and B, the ReHo value in the left MTG showed significantly positive correlation with HAMA scores: $r=0.488, P=0.005$. In this study, no other significant correlations were found between the ReHo values in regions showing significant differences and other clinical variables.

Table I Demographic and clinical characteristics of GAD patients and $\mathrm{HC}$

\begin{tabular}{lllll}
\hline Characteristics & $\begin{array}{l}\text { GAD } \\
(\mathbf{n}=\mathbf{3} \mathbf{1})\end{array}$ & $\begin{array}{l}\text { HC } \\
(\mathbf{n}=\mathbf{3 6})\end{array}$ & t-value & P-value \\
\hline Sex (male/female) & $15 / 16$ & $13 / 23$ & NA & 0.3100 \\
Age (years) & $36.9 \pm 9.2$ & $39.5 \pm 8.8$ & -1.2070 & 0.2320 \\
Illness duration (years) & $2.8 \pm 3.8$ & NA & NA & NA \\
Education (years) & $10.5 \pm 3.9$ & $11.9 \pm 3.0$ & -1.7350 & 0.0880 \\
HAMA & $22.3 \pm 5.3$ & $1.1 \pm 1.9$ & 21.1380 & 0.0000 \\
HAMD-24 & $14.9 \pm 3.3$ & $0.4 \pm 1.2$ & 23.3980 & 0.0000 \\
STAI-T & $48.9 \pm 9.1$ & $21.2 \pm 2.8$ & 16.2870 & 0.0000 \\
\hline
\end{tabular}

Note: Data are reported as mean \pm SD.

Abbreviations: GAD, generalized anxiety disorder; HAMA, Hamilton Anxiety Rating Scale; HAMD-24, Hamilton Depression Rating Scale-24 items; HC, healthy controls; NA, not available; STAI-T, State-Trait Anxiety Scale-Trait Scale.
Table 2 Brain regions showing abnormal ReHo in GAD patients in comparison to $\mathrm{HC}$

\begin{tabular}{|c|c|c|c|c|c|}
\hline \multirow[t]{2}{*}{ Brain regions } & \multirow[t]{2}{*}{ Voxels } & \multicolumn{3}{|c|}{$\begin{array}{l}\text { MNI } \\
\text { coordinates } \\
(\mathrm{mm})\end{array}$} & \multirow[t]{2}{*}{$\begin{array}{l}\text { Peak } \\
t \text {-value }\end{array}$} \\
\hline & & $\mathbf{x}$ & y & $\mathbf{z}$ & \\
\hline \multicolumn{6}{|l|}{ Increased ReHo (GAD > HC) } \\
\hline Left middle temporal gyrus & 57 & -54 & -3 & -24 & 3.7628 \\
\hline Left superior temporal gyrus & 99 & -51 & 6 & 0 & 3.4414 \\
\hline Right superior occipital gyrus & 67 & 30 & -81 & 42 & 3.0177 \\
\hline \multicolumn{6}{|l|}{ Decreased ReHo (GAD < HC) } \\
\hline Right orbital middle frontal gyrus & 82 & 12 & 60 & -9 & -3.6965 \\
\hline Left anterior cingulate cortex & 56 & -9 & 48 & 12 & -3.2629 \\
\hline Right middle frontal gyrus & 87 & 39 & 42 & 15 & -4.1522 \\
\hline Right supplementary motor area & 76 & 9 & 21 & 45 & -3.5626 \\
\hline Left supplementary motor area & 87 & -3 & 24 & 48 & -3.2133 \\
\hline
\end{tabular}

Abbreviations: GAD, generalized anxiety disorder; $\mathrm{HC}$, healthy controls; MNI, Montreal Neurological Institute; ReHo, regional homogeneity.

\section{Discussion}

Our study used ReHo to investigate the synchrony of regional spontaneous activity in rs-fMRI among patients with GAD. We found that patients with GAD showed significantly lower ReHo in the right orbital MFG, left ACC, right MFG, and bilateral SMA, as well as higher ReHo in the left MTG, left STG, and right SOG. The ReHo value of the left MTG had a significant positive correlation with the HAMA score.

The decreased ReHo values in the right orbital MFG and right MFG in the GAD group reflect destruction of local synchronization of spontaneous low-frequency fluctuations dependent on blood oxygenation levels in these two regions. This finding implies functional deficits. Frontal lobe dysfunction is characterized clinically by decrease of spontaneity, initiative, insight, judgment, abstraction, perseverance, and response inhibition. Previous neuroimaging studies showed that PFC played an important role in anxiety. Kim et $\mathrm{al}^{24}$ found a variety of anxiety disorders were typically characterized by hyperactivity of the amygdala and hypoactivity of the PFC. The disrupted emotional and cognitive processes were thought to be a crucial component of symptomatology in the pathology of anxiety.

In addition, previous studies found abnormal activation in the MFG of GAD patients. Blair et $\mathrm{al}^{25}$ reported that GAD patients had significantly increased response to angry expressions in a lateral region of the MFG, as compared to healthy individuals. This finding also related to self-reported anxiety in patients with GAD. Using 99mTchexamethylpropyleneamine oxime single-photon emission computed tomography, Kalk et al ${ }^{26}$ found increased perfusion 


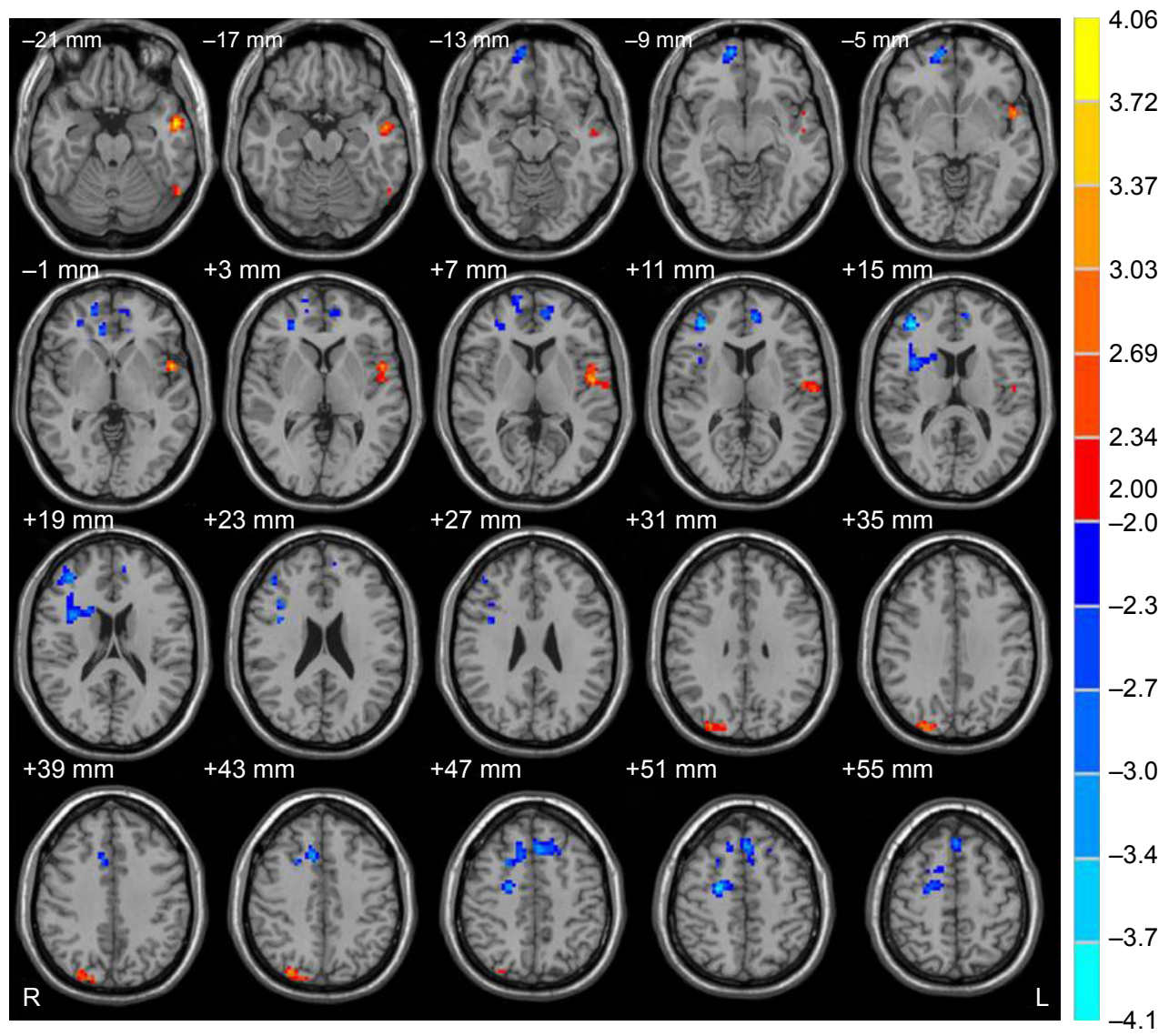

Figure I Brain regions showing abnormal ReHo in GAD patients in comparison to healthy controls.

Notes: The warm colors (positive value) represent increased ReHo areas and the cold colors (negative value) represent decreased ReHo areas. A T-score bar is shown on the right. Compared to the healthy controls, GAD patients had significantly decreased ReHo in the right orbital middle frontal gyrus, left anterior cingulate cortex, right middle frontal gyrus, and bilateral supplementary motor area. GAD patients demonstrated increased ReHo values in the left middle temporal gyrus, left superior temporal gyrus, and right superior occipital gyrus.

Abbreviations: GAD, generalized anxiety disorders; ReHo, regional homogeneity.

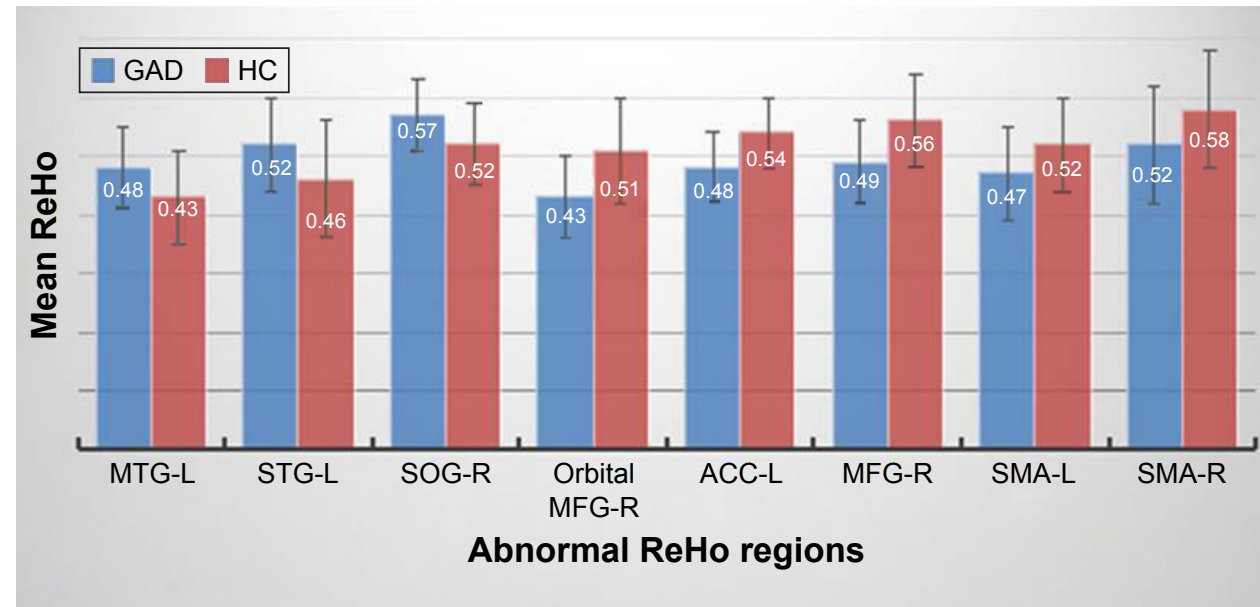

Figure 2 Bars of the mean ReHo values for the identified brain regions.

Note: All regions showed significant differences between GAD patients and healthy controls.

Abbreviations: ACC, anterior cingulate cortex; GAD, generalized anxiety disorders; HC, healthy controls; L, left; MFG, middle frontal gyrus; MTG, middle temporal gyrus;

R, right; ReHo, regional homogeneity; SMA, supplementary motor area; SOG, superior occipital gyrus; STG, superior temporal gyrus. 
A

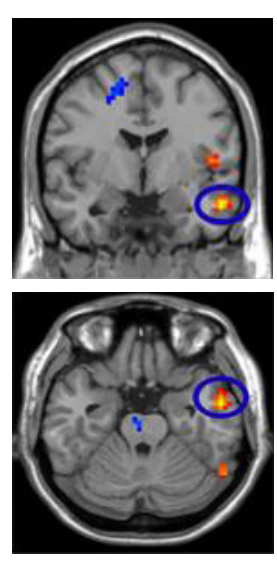

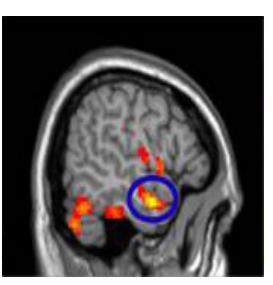

MTG-L

$(-54-3-24)$

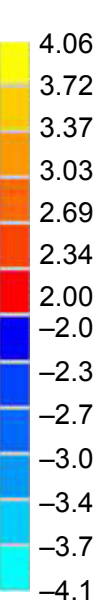

B

The correlation between HAMA and MTG-L

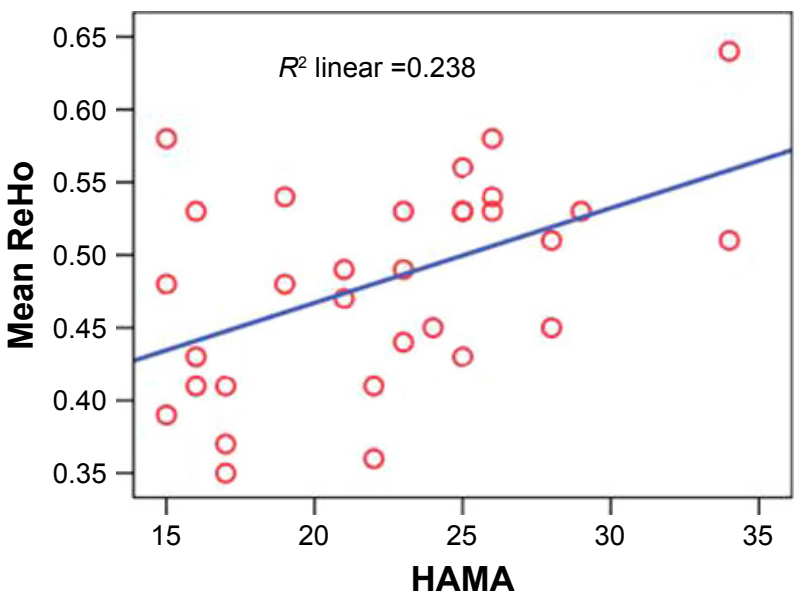

Figure 3 Correlation between MTG-L and HAMA scores.

Note: (A, B) The ReHo value of the MTG-L correlated positively with HAMA scores.

Abbreviations: HAMA, Hamilton Anxiety Rating Scale; L, left; MTG, middle temporal gyrus; ReHo, regional homogeneity.

in the right MFG in untreated GAD patients. Together with previous neuroimaging studies related to GAD, our study suggested that functional disturbance in the PFC might be a direct neural mechanism of GAD.

In addition, GAD patients showed decreased ReHo in the left ACC. The ACC is a critical component of the limbic system that has been confirmed as having a widely structural and functional connection in the PFC. ${ }^{27}$ The ACC plays an important role in emotion regulation and autonomic control, ${ }^{28}$ and the ventral ACC is involved in the elimination and adjustment of negative emotion. ${ }^{29}$ Previous fMRI studies have shown that the ACC is activated while GAD patients perform the emotional arousal task ${ }^{30}$ and it decreases while the patients perform the conflict mission and emotional face recognition tasks. ${ }^{31,32}$ Moreover, a systematic review showed GAD has been linked to hypofunction in the PFC and ACC that is associated with emotion regulation. ${ }^{33}$ The emotion dysregulation theory showed that patients with GAD experience emotional hyperarousal, contributing to maladaptive emotion regulation and unsuccessful attempts to either minimize or over-control emotions. ${ }^{34}$ Consequently, in our study, the decreased ReHo in the ACC suggests dysfunction of spontaneous neural activity, which may relate to the emotional disturbance of uncontrollable anxiety and fear in patients with GAD.

We also found decreased ReHo in the bilateral SMA, the important knot of the motor system. The SMA is tightly connected with the motor cortex and is thought to be involved in planning of motor action and cognitive control. ${ }^{35,36}$ One fMRI study ${ }^{37}$ used psychophysiologic interaction analysis to show that when patients with GAD performed the generalization task, they exhibited enhancement of anterior insula coupling with SMA. Therefore, we suggest that decreased ReHo of the SMA in our study may be related to the impairment of the motor system, muscular tension, and motor restlessness induced by the loss of inhibition reaction in GAD patients.

Our results showed that patients with GAD exhibited increased ReHo in the left STG. Quirk et $\mathrm{al}^{38}$ indicated that the STG was associated with senior cognitive processes with regard to scary experience of humans, as well as the modulation function to activity in the amygdala. Roy et $\mathrm{al}^{39}$ found that the functional connectivities between the amygdala with STG and insula were positively correlated with the anxiety severity scores in the GAD group. In addition, previous studies indicated that the STG in patients with anxiety disorders such as GAD, panic disorder, and social anxiety disorder was atrophied, compared with the control group. The insula and STG play an important role in regulating interoception, which is the sensation of the physiologic condition from the internal organs, such as temperature, pain, and movement, induced by an anxiety response. ${ }^{40}$ Using voxel-based morphometry, Moon et $\mathrm{al}^{41}$ found reduced volumes of the insula and STG in GAD patients. These findings suggested that the morphologic alterations of the insula and STG were associated with difficulties in emotional response and lower function of interoceptive sense caused by the anxiety symptoms of GAD. Based on the previous findings, we suppose that increased spontaneous neural activity in STG patients with GAD was a compensatory response against anxiety reaction. 
We also found that the left MTG showed increased ReHo value. The MTG is involved in many functions, such as language processing, observation of motion, deductive reasoning, and dynamic facial expressions. ${ }^{42-44}$ Engels et a ${ }^{45}$ showed activation in the left MTG for anxious apprehension using an emotional Stroop task-based fMRI. Using an automated surface-based approach (FreeSurfer), Strawn et al ${ }^{46}$ found increased cortical thickness in the left inferior and middle temporal cortex in youth with GAD. The involvement of the middle and inferior temporal gyri in emotional processing was supported by anatomic studies indicating that the posterior temporal cortex received input from visual sensory cortices ${ }^{47}$ and directed back projections from the amygdala. ${ }^{48}$ In our study, we found significant positive correlation between the ReHo value in the left MTG and the HAMA score. The more severe the level of anxiety in patients with GAD, the more they demonstrated overt spontaneous activity in the MTG that might involve implicit emotion processing as a compensatory response against anxiety.

Interestingly, we found increased ReHo value in the right SOG. The occipital lobe is the visual information processing center and is responsible for part of language, action feeling, and abstract concepts. A previous study using structural MRI found increased cortical thickness in the occipital region in GAD patients compared to healthy subjects. The finding pointed to the occipital cortex as a region of brain associated with the pathology of and clinical improvement in GAD. ${ }^{49}$ Other previous studies found that lateral occipital cortical thickness in adolescents with GAD participates in retrograde modulation of the amygdala. ${ }^{50,51}$ In pediatric patients with GAD, Strawn et $\mathrm{al}^{46}$ also found increased cortical thickness in the right lateral occipital cortex. Furthermore, patients with GAD showed significantly lower activities in the SOG during the use of neutral and anxiety-inducing activities, which can be considered relatively lower ability for visual information processing and deficits of visual attention, compared to healthy controls. ${ }^{52}$ Considering these previous studies, the increased $\mathrm{ReHo}$ in the right SOG in our study might be a compensatory response involving disorders of emotion regulation.

It is noteworthy that there are still several limitations in this study. First, the number of subjects in our study was relatively small, which could affect the statistical analysis and comprehensive interpretation of the findings. A further study including more datasets is needed. Second, although we found abnormalities in the motor cortex, we did not test the motor-related scales. It would be better for future research to use motor-related scales to investigate more fully the decreased spontaneous activity in the motor cortex in patients with GAD. Third, this study is a cross-section preliminary study, and the findings of the study cannot provide longitudinal alteration data on GAD patients.

\section{Conclusion}

In conclusion, our results showed altered synchrony of regional spontaneous activity in GAD patients based on rs-fMRI data. The results indicated that altered functional activities are mainly located in the emotional sensorimotor and vision-related regions. In addition, the spontaneous regional activities in the anterior cingulate gyrus, frontal lobe, and temporal lobe may be closely related to the neural mechanism and clinical manifestation of GAD.

\section{Acknowledgments}

The authors thank Professor Ruiwang Huang at the South China Normal University for the technical support, Xiaoyan $\mathrm{Pu}$ at the People's Hospital of Yuxi City for data collection, and all the reviewers who improved the article greatly. This work was supported by funding from the National Natural Science Foundation of China (Grant number: 81471639), the Natural Science Foundation of Guangdong Province, China (Grant number: 2015A030313723), and the Science and Technology Planning Project of Guangzhou City, China (Grant number: 201607010056).

We declare that our work described here has not been submitted elsewhere for publication, either in whole or in part.

\section{Author contributions}

All authors of this research paper have directly participated in the planning, execution, or analysis of this study. All authors contributed toward data analysis, drafting and revising the paper and agree to be accountable for all aspects of the work.

\section{Disclosure}

The authors report no conflicts of interest in this work.

\section{References}

1. Wittchen HU, Jacobi F, Rehm J, et al. The size and burden of mental disorders and other disorders of the brain in Europe 2010. Eur Neuropsychopharmacol. 2011;21(9):655-679.

2. Wittchen HU, Jacobi F. Size and burden of mental disorders in Europe - a critical review and appraisal of 27 studies. Eur Neuropsychopharmacol. 2005;15(4):357-376.

3. Bernal M, Haro JM, Bernert S, et al. Risk factors for suicidality in Europe: results from the ESEMED study. J Affect Disord. 2007;101(1-3): 27-34.

4. Lee LT, Tsai HC, Chi MH, et al. Lower availability of striatal dopamine transporter in generalized anxiety disorder: a preliminary two-ligand SPECT study. Int Clin Psychopharmacol. 2015;30(3):175-178. 
5. Moon CM, Kang HK, Jeong GW. Metabolic change in the right dorsolateral prefrontal cortex and its correlation with symptom severity in patients with generalized anxiety disorder: proton magnetic resonance spectroscopy at 3 Tesla. Psychiatry Clin Neurosci. 2015; 69(7):422-430.

6. Hettema JM, Kettenmann B, Ahluwalia V, et al. Pilot multimodal twin imaging study of generalized anxiety disorder. Depress Anxiety. 2012;29(3):202-209.

7. Tromp DP, Grupe DW, Oathes DJ, et al. Reduced structural connectivity of a major frontolimbic pathway in generalized anxiety disorder. Arch Gen Psychiatry. 2012;69(9):925-934.

8. McClure EB, Monk CS, Nelson EE, et al. Abnormal attention modulation of fear circuit function in pediatric generalized anxiety disorder. Arch Gen Psychiatry. 2007;64(1):97-106.

9. Monk CS, Telzer EH, Mogg K, et al. Amygdala and ventrolateral prefrontal cortex activation to masked angry faces in children and adolescents with generalized anxiety disorder. Arch Gen Psychiatry. 2008;65(5):568-576.

10. Yassa MA, Hazlett RL, Stark CE, Hoehn-Saric R. Functional MRI of the amygdala and bed nucleus of the stria terminalis during conditions of uncertainty in generalized anxiety disorder. J Psychiatr Res. 2012; 46(8): 1045-1052.

11. Ball TM, Ramsawh HJ, Campbell-Sills L, Paulus MP, Stein MB. Prefrontal dysfunction during emotion regulation in generalized anxiety and panic disorders. Psychol Med. 2013;43(7):1475-1486.

12. Peterson A, Thome J, Frewen P, Lanius RA. Resting-state neuroimaging studies: a new way of identifying differences and similarities among the anxiety disorders? Can J Psychiatry. 2014;59(6):294-300.

13. Li W, Cui H, Zhu Z, et al. Aberrant functional connectivity between the amygdala and the temporal pole in drug-free generalized anxiety disorder. Front Hum Neurosci. 2016;10:549.

14. Makovac E, Watson DR, Meeten F, et al. Amygdala functional connectivity as a longitudinal biomarker of symptom changes in generalized anxiety. Soc Cogn Affect Neurosci. 2016;11(11):1719-1728.

15. Rabany L, Diefenbach GJ, Bragdon LB, et al. Resting-state functional connectivity in generalized anxiety disorder and social anxiety disorder: evidence for a dimensional approach. Brain Connect. 2017;7(5): 289-298.

16. Dai XJ, Gong HH, Wang YX, et al. Gender differences in brain regional homogeneity of healthy subjects after normal sleep and after sleep deprivation: a resting-state fMRI study. Sleep Med. 2012;13(6): $720-727$.

17. Guo J, Chen N, Li R, et al. Regional homogeneity abnormalities in patients with transient ischaemic attack: a resting-state fMRI study. Clin Neurophysiol. 2014;125(3):520-525.

18. Tang YL, Ji GJ, Yu Y, et al. Altered regional homogeneity in rolandic epilepsy: a resting-state FMRI study. Biomed Res Int. 2014;2014: 960395.

19. Wu QZ, Li DM, Kuang WH, et al. Abnormal regional spontaneous neural activity in treatment-refractory depression revealed by restingstate fMRI. Hum Brain Mapp. 2011;32(8):1290-1299.

20. Chao-Gan Y, Yu-Feng Z. DPARSF: a MATLAB toolbox for "pipeline" data analysis of resting-state fMRI. Front Syst Neurosci. 2010;4:13.

21. Biswal B, Yetkin FZ, Haughton VM, Hyde JS. Functional connectivity in the motor cortex of resting human brain using echo-planar MRI. Magn Reson Med. 1995;34(4):537-541.

22. Wu T, Long X, Zang Y, et al. Regional homogeneity changes in patients with Parkinson's disease. Hum Brain Mapp. 2009;30(5): $1502-1510$

23. Zang Y, Jiang T, Lu Y, He Y, Tian L. Regional homogeneity approach to fMRI data analysis. Neuroimage. 2004;22(1):394-400.

24. Kim MJ, Loucks RA, Palmer AL, et al. The structural and functional connectivity of the amygdala: from normal emotion to pathological anxiety. Behav Brain Res. 2011;223(2):403-410.

25. Blair K, Shaywitz J, Smith BW, et al. Response to emotional expressions in generalized social phobia and generalized anxiety disorder: evidence for separate disorders. Am J Psychiatry. 2008;165(9):1193-1202.
26. Kalk NJ, Melichar J, Holmes RB, et al. Central noradrenergic responsiveness to a clonidine challenge in generalized anxiety disorder: a single photon emission computed tomography study. J Psychopharmacol. 2012;26(4):452-460.

27. Stevens FL, Hurley RA, Taber KH. Anterior cingulate cortex: unique role in cognition and emotion. J Neuropsychiatry Clin Neurosci. 2011;23(2): $121-125$.

28. Critchley HD, Mathias CJ, Josephs O, et al. Human cingulate cortex and autonomic control: converging neuroimaging and clinical evidence. Brain. 2003;126(Pt 10):2139-2152.

29. Schiller D, Delgado MR. Overlapping neural systems mediating extinction, reversal and regulation of fear. Trends Cogn Sci. 2010;14(6): 268-276.

30. Paulesu E, Sambugaro E, Torti T, et al. Neural correlates of worry in generalized anxiety disorder and in normal controls: a functional MRI study. Psychol Med. 2010;40(1):117-124.

31. Blair KS, Geraci M, Smith BW, et al. Reduced dorsal anterior cingulate cortical activity during emotional regulation and top-down attentional control in generalized social phobia, generalized anxiety disorder, and comorbid generalized social phobia/generalized anxiety disorder. Biol Psychiatry. 2012;72(6):476-482

32. Palm ME, Elliott R, McKie S, Deakin JF, Anderson IM. Attenuated responses to emotional expressions in women with generalized anxiety disorder. Psychol Med. 2011;41(5):1009-1018.

33. Brooks SJ, Stein DJ. A systematic review of the neural bases of psychotherapy for anxiety and related disorders. Dialogues Clin Neurosci. 2015;17(3):261-279.

34. Ball TM, Stein MB, Paulus MP. Toward the application of functional neuroimaging to individualized treatment for anxiety and depression. Depress Anxiety. 2014;31(11):920-933.

35. Carson RG. Neural pathways mediating bilateral interactions between the upper limbs. Brain Res Brain Res Rev. 2005;49(3):641-662.

36. Nachev P, Kennard C, Husain M. Functional role of the supplementary and pre-supplementary motor areas. Nat Rev Neurosci. 2008;9(11): 856-869.

37. Greenberg T, Carlson JM, Cha J, Hajcak G, Mujica-Parodi LR. Ventromedial prefrontal cortex reactivity is altered in generalized anxiety disorder during fear generalization. Depress Anxiety. 2013;30(3): $242-250$.

38. Quirk GJ, Armony JL, LeDoux JE. Fear conditioning enhances different temporal components of tone-evoked spike trains in auditory cortex and lateral amygdala. Neuron. 1997;19(3):613-624.

39. Roy AK, Fudge JL, Kelly C, et al. Intrinsic functional connectivity of amygdala-based networks in adolescent generalized anxiety disorder. J Am Acad Child Adolesc Psychiatry. 2013;52(3):290-299.

40. van Tol MJ, van der Wee NJ, van den Heuvel OA, et al. Regional brain volume in depression and anxiety disorders. Arch Gen Psychiatry. 2010; 67(10):1002-1011.

41. Moon CM, Kim GW, Jeong GW. Whole-brain gray matter volume abnormalities in patients with generalized anxiety disorder: voxel-based morphometry. Neuroreport. 2014;25(3):184-189.

42. Fiebach CJ, Friederici AD, Smith EE, Swinney D. Lateral inferotemporal cortex maintains conceptual-semantic representations in verbal working memory. J Cogn Neurosci. 2007;19(12):2035-2049.

43. Tyler LK, Bright P, Dick E, et al. Do semantic categories activate distinct cortical regions? Evidence for a distributed neural semantic system. Cogn Neuropsychol. 2003;20(3):541-559.

44. Xu J, Wang J, Fan L, et al. Tractography-based Parcellation of the Human Middle Temporal Gyrus. Sci Rep. 2015;5:18883.

45. Engels AS, Heller W, Mohanty A, et al. Specificity of regional brain activity in anxiety types during emotion processing. Psychophysiology. 2007;44(3):352-363.

46. Strawn JR, John WC, Dominick KC, et al. Cortical surface anatomy in pediatric patients with generalized anxiety disorder. J Anxiety Disord. 2014;28(7):717-723.

47. Ungerleider LG, Haxby JV. 'What' and 'where' in the human brain. Curr Opin Neurobiol. 1994;4(2):157-165. 
48. Amaral DG. The primate amygdala and the neurobiology of social behavior: implications for understanding social anxiety. Biol Psychiatry. 2002;51(1):11-17.

49. Abdallah CG, Coplan JD, Jackowski A, et al. Riluzole effect on occipital cortex: a structural and spectroscopy pilot study. Neurosci Lett. 2012;530(1):103-107.

50. Morris JS, Friston KJ, Buchel C, et al. A neuromodulatory role for the human amygdala in processing emotional facial expressions. Brain. 1998;121(Pt 1):47-57.
51. Pessoa L, McKenna M, Gutierrez E, Ungerleider LG. Neural processing of emotional faces requires attention. Proc Natl Acad Sci US A. 2002; 99(17):11458-11463.

52. Moon CM, Jeong GW. Functional neuroanatomy on the working memory under emotional distraction in patients with generalized anxiety disorder. Psychiatry Clin Neurosci. 2015;69(10):609-619.

\section{Publish your work in this journal}

Neuropsychiatric Disease and Treatment is an international, peerreviewed journal of clinical therapeutics and pharmacology focusing on concise rapid reporting of clinical or pre-clinical studies on a range of neuropsychiatric and neurological disorders. This journal is indexed on PubMed Central, the 'PsycINFO' database and CAS, and is the official journal of The International Neuropsychiatric Association (INA). The manuscript management system is completely online and includes a very quick and fair peer-review system, which is all easy to use. Visit http://www.dovepress.com/testimonials.php to read real quotes from published authors.

Submit your manuscript here: http://www.dovepress.com/neuropsychiatric-disease-and-treatment-journal 